\title{
High Risk of Venous Thrombosis Recurrence in Fully Anticoagulated Patient with Antithrombin Deficiency during COVID-19: A Case Report
}

\author{
Mirjana Kovac ${ }^{1,2}$ Olivera Markovic ${ }^{3} \quad$ Sanja Lalic-Cosic ${ }^{4}$ Gorana Mitic $^{5}$
}

${ }^{1}$ Faculty of Medicine, University of Belgrade, Belgrade, Serbia

${ }^{2}$ Department of Hemostasis, Blood Transfusion Institute of Serbia, Belgrade, Serbia

${ }^{3}$ Department of Hematology, University Hospital Medical Center Bezanijska Kosa, Belgrade, Serbia

${ }^{4}$ Faculty of Pharmacy, University of Belgrade, Belgrade, Serbia

${ }^{5}$ Center of Laboratory Medicine, Clinical Center of Vojvodina, Faculty of Medicine Novi Sad, University of Novi Sad, Novi Sad, Serbia
Address for correspondence Mirjana Kovac, Department of Hemostasis, Blood Transfusion Institute of Serbia, Sv. Save 39, 11000 Belgrade, Serbia (e-mail: mkovac008@gmail.com).

Ind J Car Dis Wom 2021;6:252-255.

\begin{abstract}
Coagulation dysfunction is a serious issue in patients with Coronavirus disease-19 (COVID-19). With regard to recently published studies, a high number of patients with acute respiratory distress syndrome (ARDS) secondary to COVID-19 developed lifethreatening thrombotic complications despite anticoagulation. We report a case of young woman with the type-II heparin-binding site (HBS) antithrombin (AT) deficiency (Budapest 3-homozygous), who developed acute deep vein thrombosis on two occasions due to COVID-19 infection in the course of stable anticoagulation with

Keywords

- acute deep venous thrombosis

- recurrent thrombosis

- antithrombin deficiency

- type-II HBS

- COVID-19 vitamin $\mathrm{K}$ antagonist. The first thrombotic event was observed during mild COVID-19 infection, while the second thrombotic event she developed 2 months after she was negative for severe acute respiratory syndrome Coronavirus-2 (SARS-CoV-2). Our case highlights the complexity of the treatment in this particular type of thrombophilia and the need for precaution even in mild forms of viral infection. In the treatment of acute thrombosis, AT-deficient patients may benefit from the use of AT concentrate along with low-molecular weight heparin (LMWH), while in cases of type II-HBS, AT supplementation is mandatory.
\end{abstract}

\section{Introduction}

The clinical course of Coronavirus disease-19 (COVID-19) infection varies from asymptomatic, mild symptoms, severe illness and sepsis to death. ${ }^{1,2}$ Coagulation dysfunction is a serious complication in patients with COVID-19, whereby fulminant thrombotic complications emerge as critical issues in those with severe COVID-19. ${ }^{3}$ There are several changes in the prothrombotic direction, which can be explained by the profound inflammatory response as well as by hypoxia. ${ }^{1,2}$ Significant activation of the coagulation system caused by this novel Coronavirus may result in vascular complications, mainly venous thromboembolism (VTE), which have significant implications on the clinical published online December 14, 2021
DOI https://doi.org/

10.1055/s-0041-1739129. ISSN 2455-7854.

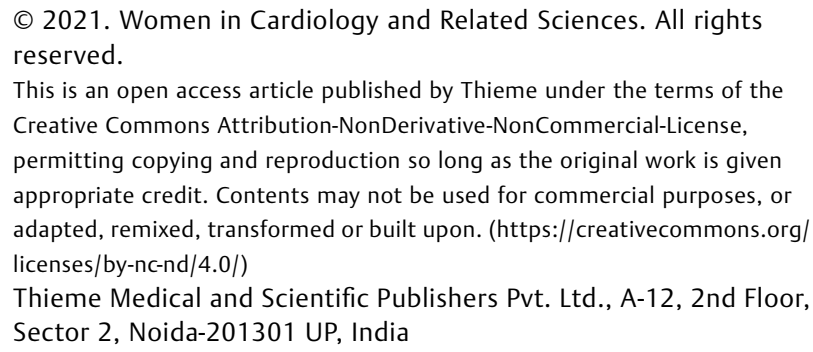

This is an open access article published by Thieme under the terms of the Creative Commons Attribution-NonDerivative-NonCommercial-License, permitting copying and reproduction so long as the original work is given appropriate credit. Contents may not be used for commercial purposes, or adapted, remixed, transformed or built upon. (https://creativecommons.org/ licenses/by-nc-nd/4.0/)

Thieme Medical and Scientific Publishers Pvt. Ltd., A-12, 2nd Floor, Sector 2, Noida-201301 UP, India 
outcome for COVID-19 patients. ${ }^{4}$ Thus, many patients with acute respiratory distress syndrome (ARDS) secondary to COVID-19 developed life-threatening thrombotic complications despite anticoagulation. ${ }^{5}$ Additionally, inherited thrombophilia might contribute to the increased risk of VTE during COVID-19 infection. However, there is very little data on association of congenital thrombophilia and thrombotic events during COVID infection. A recently published study that included 13 patients with congenital thrombophilia showed that most patients with severe thrombophilia did not develop symptomatic thrombotic events during COVID-19, including those who had previous thromboses. They state that the possible explanation for the low incidence rate of thrombosis in thrombophilia patients may be related to the fact that these patients were already treated with anticoagulant drugs before the infection or at the very early stages of the disease. ${ }^{6}$ Previous findings ${ }^{3}$ also support that long-term anticoagulation at admission appears to protect COVID-19 patients from VTE.

\section{Case Details}

Here, we report the case of a young woman carrier of severe thrombophilia, type-II heparin-binding site (HBS) antithrombin (AT) deficiency (Budapest 3-homozygous), who developed acute deep vein thrombosis (DVT) in two occasions in the course of stable anticoagulation with vitamin $\mathrm{K}$ antagonist (VKA). The first thrombotic event was observed during mild COVID-19 infection, while the second thrombotic event developed 2 months after she was negative for severe acute respiratory syndrome Coronavirus-2 (SARS-CoV-2).

A young woman aged 32 was admitted in a COVID hospital due to development of respiratory symptoms, mild rhinitis and sore throat without fever. She was not a smoker, her body mass index (BMI) of 22.4 was in the normal range but with pronounced varicose veins in both legs. Previously, due to the development of spontaneous recurrent juvenile thrombosis (proximal DVT affecting both legs), she was diagnosed as an AT-deficient patient at the age of 14 years. She was subjected to long-term VKA. The use of VKA therapy was regularly monitored to maintain the therapeutic range of international normalized ratio (INR) between 2.5 and 3.5. During the last 6 months prior to COVID infection, she was on stable anticoagulation treatment, and the last control in early June showed an INR of 2.95 .

In early July 2020, she contacted the anticoagulation service, asking for advice since her partner was found to be polymerase chain reaction (PCR)-positive for COVID-19. Given the severity of thrombophilia in her case and the effect of the COVID-19 infection on the development of thrombotic complications, she was advised to report to the COVID hospital immediately for testing and examination. Due to the development of respiratory symptoms and her thrombophilia status, she spent the next 7 days in the COVID hospital. Her nasopharyngeal swab SARS-CoV-2 reverse transcriptase-polymerase chain reaction (RT-PCR) test was positive on July 8,2020 , while a chest X-ray showed initial signs of bilateral pneumonia, and she was given standard therapy for
COVID-19 treatment, including an antibiotic (azithromycin), vitamins and continuation of the VKA with the hematologist's recommendation that the INR level should be maintained around 3. She was afebrile at all times in a stable general condition with no need for any respiratory support. As someone with mild symptoms, she was discharged on July 15, 2020, in good condition with normal biochemical and hematological parameters (-Table $\mathbf{1}$ ) for home treatment, with advice to rest and continue the prescribed therapy.

A week after discharge from the hospital, she experienced a weird feeling in her right leg in an upright position but did not report any swelling. She was referred to the COVID hospital immediately for an examination due to suspected acute thrombosis. On admission to the hospital on July 23, 2020, an INR of 2.21 and D-dimer of $4.22 \mathrm{mg} / \mathrm{L}$ fibrinogen equivalent units (FEU) $(0.5 \mathrm{mg} / \mathrm{L}$ cutoff value used for discrimination of VTE) were obtained. The ultrasound examination showed the presence of an acute thrombotic process with subocclusive thrombotic mass (5-7 cm long), affecting popliteal and tibial veins of the lower right leg. The vascular surgeon switched anticoagulant therapy from VKA to therapeutic doses of low molecular weight heparin (LMWH) and nadroparin 5700 IU subcutaneously (SC) twice daily. In consultation with the hematologist, AT concentrate was introduced. The first administered dose of AT III concentrate (Kybernin) was $3000 \mathrm{IU}$ followed by $1500 \mathrm{IU}$ in the next 5 days. An AT activity range of 80 to $100 \%$ was maintained with monitoring. After clinical improvement, VKA was reintroduced, overlapping LMWH until the therapeutic range of INR was reached. She was discharged on August 3, 2020, on stable anticoagulation, while her nasopharyngeal swab SARS-CoV-2 RT-PCR test was negative.

During next few weeks, stability of the anticoagulant therapy was confirmed by INR 3.0 and D-dimer $0.92 \mathrm{mg} / \mathrm{L}$ FEU, while AT activity at $12 \%$ (ref. range $83-113 \%$ ) and factor VIII (FVIII) level of $2.5 \mathrm{IU} / \mathrm{L}$ (ref. range $0.50-1.50 \mathrm{IU} / \mathrm{L}$ ) were obtained. The patient complained of fatigue and malaise. Two months after she become PCR negative for SARS-CoV-2, she reported pain in the right leg again. The ultrasound examination showed the presence of a new thrombotic event in the area of the gastrocnemius (solei veins). She was hospitalized again on September 3, 2020, with continued VKA therapy, maintenance of the INR level $>3$, and inclusion of AT concentrate substitution at an initial dose of $3000 \mathrm{IU}$ followed by 2000 IU daily. On September 9, 2020, she was discharged with stable anticoagulation (INR 3.8) and an AT level of 66\%. For the next 6 months, the INR was monitored weekly with the aim of maintaining over 3. Factor VIII and AT activity were monitored monthly. In the following months, elevated levels of FVIII of $2.5 \mathrm{IU} / \mathrm{L}$ were observed, while AT activity of $45 \%$ was similar to the previously established AT level, which was obtained during the thrombophilia examination after her first DVT.

\section{Discussion}

As far as we know, this is the first case of recurrent acute DVT diagnosed during COVID-19 infection in a fully 
Table 1 Biochemical, hematological and hemostatic parameters

\begin{tabular}{|c|c|c|c|c|}
\hline & \multicolumn{2}{|c|}{$\begin{array}{l}\text { On discharge during the first } \\
\text { hospitalization } \\
15 / 07 / 2020\end{array}$} & $\begin{array}{l}\text { On discharge during } \\
\text { the second hospitalization } \\
03 / 08 / 2020\end{array}$ & $\begin{array}{l}\text { On discharge during } \\
\text { the third hospitalization } \\
09 / 09 / 2020\end{array}$ \\
\hline \multicolumn{5}{|l|}{ Biochemical parameters } \\
\hline Glucose (mmol/L) & \multicolumn{2}{|l|}{4.6} & 4.4 & 4.6 \\
\hline Urea (mmol/L) & \multicolumn{2}{|l|}{3.6} & 2.9 & 4.3 \\
\hline Creatinine (umol/L) & \multicolumn{2}{|l|}{65} & 52 & 56 \\
\hline Total bilirubin (umol/L) & \multicolumn{2}{|l|}{7.1} & 4.7 & 7.3 \\
\hline Direct bilirubin (umol/L) & \multicolumn{2}{|l|}{2} & 1.9 & 2.3 \\
\hline AST (U/L) & \multicolumn{2}{|l|}{18} & 15 & 18 \\
\hline $\operatorname{ALT}(\mathrm{U} / \mathrm{L})$ & \multicolumn{2}{|l|}{15} & 13 & 13 \\
\hline $\mathrm{LDH}(\mathrm{U} / \mathrm{L})$ & \multicolumn{2}{|l|}{337} & 308 & 380 \\
\hline CK $(\mathrm{U} / \mathrm{L})$ & \multicolumn{2}{|l|}{88} & 0.7 & 236 \\
\hline Total protein $(\mathrm{g} / \mathrm{L})$ & \multicolumn{2}{|l|}{77} & 61 & 64 \\
\hline Albumin $(\mathrm{g} / \mathrm{L})$ & \multicolumn{2}{|l|}{44} & 44 & 44 \\
\hline CRP (mg/L) & \multicolumn{2}{|l|}{1.5} & 0.7 & 0.6 \\
\hline Ferritin (ug/L) & \multicolumn{2}{|l|}{28} & 84 & 34 \\
\hline \multicolumn{5}{|l|}{ Hematological parameters } \\
\hline WBC $\left(10^{9} / \mathrm{L}\right)$ & \multicolumn{2}{|l|}{3.42} & 3.56 & 4.20 \\
\hline NEUT (\%) & \multicolumn{2}{|l|}{58.1} & 56.1 & 50.0 \\
\hline LYMPH (\%) & \multicolumn{2}{|l|}{31.6} & 33.6 & 41.0 \\
\hline MONO (\%) & \multicolumn{2}{|l|}{9.1} & 9.2 & 6.8 \\
\hline $\mathrm{EO}(\%)$ & \multicolumn{2}{|l|}{0.9} & 0.8 & 1.7 \\
\hline BASO (\%) & \multicolumn{2}{|l|}{0.3} & 0.3 & 0.5 \\
\hline $\operatorname{RBC}\left(10^{12} / \mathrm{L}\right)$ & \multicolumn{2}{|l|}{4.33} & 4.14 & 3.90 \\
\hline $\mathrm{HGB}(\mathrm{g} / \mathrm{L})$ & \multicolumn{2}{|l|}{131} & 123 & 119 \\
\hline $\mathrm{HCT}(\mathrm{L} / \mathrm{L})$ & \multicolumn{2}{|l|}{0.402} & - & - \\
\hline MCV (fl) & \multicolumn{2}{|l|}{92.8} & 88.9 & 90 \\
\hline $\operatorname{PLT}\left(10^{9} / \mathrm{L}\right)$ & 225 & & 215 & 214 \\
\hline Hemostatic parameters & & & & \\
\hline PT INR & 3.30 & & 2.71 & 3.60 \\
\hline APTT (s) & 33.5 & & 32.7 & 36.3 \\
\hline Fibrinogen $(\mathrm{g} / \mathrm{L})$ & 3.0 & & 2.5 & 2.8 \\
\hline D-dimer (mg/L FEU) & 0.36 & & 1.74 & 0.40 \\
\hline AT (\%) & - & & 78.6 & 65.0 \\
\hline FVIII (IU/L) & - & & - & 2.42 \\
\hline Hemostatic test results be & two hospita & ons & & \\
\hline & $13 / 08 / 2020$ & $17 / 08 / 2020$ & $21 / 08 / 2020$ & $25 / 08 / 2020$ \\
\hline PT INR & 5.40 & 2.50 & 4.50 & 3.00 \\
\hline APTT (s) & - & - & - & 36.2 \\
\hline Fibrinogen $(\mathrm{g} / \mathrm{L})$ & - & - & - & 3.6 \\
\hline D-dimer (mg/L FEU) & 0.80 & - & - & 0.92 \\
\hline AT (\%) & - & - & - & 12.0 \\
\hline FVIII (IU/L) & - & - & - & 2.50 \\
\hline
\end{tabular}

Abbreviations: AT, antithrombin; ALT, alanine transaminase; APTT, activated partial thromboplastin time; AST, aspartate transaminase; BASO, basophil; CK, creatine kinase; CRP, C-reactive protein; EO, eosinophil; FEU, fibrinogen equivalent unit; HCT, hematocrit; HGB, hemoglobin; INR, international normalized ratio; $L D H$, lactate dehydrogenase; LYMPH, lymphocyte; MCV, mean corpuscular volume; MONO, monocyte; NEUT, neutrophil; PT, prothrombin time; PLT, platelet; RBC, red blood cell; WBC, white blood cell. Innovance D-dimer (Siemens) normal range up to $0.55 \mathrm{mg} / \mathrm{L} \mathrm{FEU,} \mathrm{AT} \mathrm{Innovance} \mathrm{AT} \mathrm{(Siemens),} \mathrm{normal} \mathrm{range} 83-118 \%$. 
anticoagulated patient taking VKA, who is a carrier of severe thrombophilia, type-II HBS AT deficiency (Budapest 3 ) in the homozygous variant. Since she was a patient with mild symptoms of COVID-19 and recovered very quickly without needing any respiratory support, no AT level was measured during the first hospitalization and she was discharged with advice to rest and continue the prescribed therapy. The symptoms of acute thrombosis appeared very soon, during the first week after discharge, while the second recurrent event developed in a period 2 months after she become PCRnegative for SARS-CoV-2. In both cases, she was fully anticoagulated but with the lowest ever measured AT activity of only $12 \%$ and a steadily elevated level of FVIII, whose values were maintained in the following months.

Presently, there is a consensus that all patients admitted to hospital with COVID-19 receive prophylactic anticoagulation. ${ }^{7}$ According to existing guidelines, the use of LMWH is recommended in COVID-19-induced thrombosis. In those patients who are ready for discharge, direct oral anticoagulants (DOACs) or LMWH would be preferred to limit contact of patients with health care services required for INR monitoring for VKAs. ${ }^{7,8}$ Due to the pointed severe thrombophilia and recurrent DVT, our patient was already on stable VKA with the INR level that was maintained in the range of approximately 3 . However, considering the pathophysiological mechanism and effect of this particular type of AT deficiency in treatment for acute thrombosis, it was necessary to introduce substitution with AT concentrate together with therapeutic doses of LMWH.

Our case highlights the complexity of COVID-19 infection treatment in this particular type of thrombophilia. Although, in this case, AT levels were basically very low due to the homozygosity of the mentioned mutation, COVID-19 infection itself might cause a further decrease, ${ }^{9,10}$ as was proved during the second recurrent event, with AT activity at only $12 \%$. No less important is the finding that even 2 months after overcoming COVID-19 infection, a high concentration of FVIII was maintained. All of these can be considered as additional risk factors for recurrent events in this case.

\section{Conclusion}

AT-deficient patients are at high risk of VTE recurrence, even when the viral infection manifests with a mild clinical picture. The risk may be present for several months after COVID-19 infection, and we would like to raise awareness of this issue among physicians who are involved in the treatment of COVID-19. In the treatment of acute thrombosis, ATdeficient patients may benefit from the use of AT concentrate along with LMWH, while in cases of type II-HBS, AT supplementation is mandatory.

Conflict of Interest

None declared.

\section{References}

1 Fei Y, Tang N, Liu H, Cao W. Coagulation dysfunction. Arch Pathol Lab Med 2020;144(10):1223-1229

2 Zhou F, Yu T, Du R, et al. Clinical course and risk factors for mortality of adult inpatients with COVID-19 in Wuhan, China: a retrospective cohort study. Lancet 2020;395(10229):1054-1062

3 Klok FA, Kruip MJHA, van der Meer NJM, et al. Incidence of thrombotic complications in critically ill ICU patients with COVID-19. Thromb Res 2020;191:145-147

4 Langer F, Kluge S, Klamroth R, Oldenburg J. Coagulopathy in COVID-19 and its implication for safe and efficacious thromboprophylaxis. Hamostaseologie 2020;40:1-6

5 Helms J, Tacquard C, Severac F, et al; CRICS TRIGGERSEP Group (Clinical Research in Intensive Care and Sepsis Trial Group for Global Evaluation and Research in Sepsis) High risk of thrombosis in patients with severe SARS-CoV-2 infection: a multicenter prospective cohort study. Intensive Care Med 2020;46(06):1089-1098

6 de la Morena-Barrio ME, Bravo-Pérez C, de la Morena-Barrio B, et al. A pilot study on the impact of congenital thrombophilia in COVID-19. Eur J Clin Invest 2021;51(05):e13546

7 Bikdeli B, Madhavan MV, Jimenez D, et al; Global COVID-19 Thrombosis Collaborative Group, Endorsed by the ISTH, NATF, ESVM, and the IUA, Supported by the ESC Working Group on Pulmonary Circulation and Right Ventricular Function. COVID-19 and thrombotic or thromboembolic disease: implications for prevention, antithrombotic therapy, and follow-up. J Am Coll Cardiol 2020;75(23):2950-2973

8 Spyropoulos AC, Levy JH, Ageno W, et al; Subcommittee on Perioperative, Critical Care Thrombosis, Haemostasis of the Scientific, Standardization Committee of the International Society on Thrombosis and Haemostasis. Scientific and Standardization Committee communication: clinical guidance on the diagnosis, prevention, and treatment of venous thromboembolism in hospitalized patients with COVID-19. J Thromb Haemost 2020;18 (08):1859-1865

9 Han H, Yang L, Liu R, et al. Prominent changes in blood coagulation of patients with SARS-CoV-2 infection. Clin Chem Lab Med 2020; 58(07):1116-1120

10 Tang N, Bai H, Chen X, Gong J, Li D, Sun Z. Anticoagulant treatment is associated with decreased mortality in severe coronavirus disease 2019 patients with coagulopathy. J Thromb Haemost 2020;18(05):1094-1099 